\title{
Approximations and Indexes in the Medicine: the BMI and the BPHi
}

\author{
Istvan G Telessy* \\ Department of Pharmaceutics, University of Pecs, Hungary
}

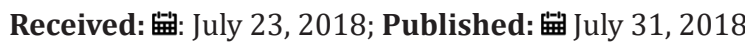

*Corresponding author: Istvan G Telessy, Department of Pharmaceutics, University of Pecs, and MedBioFit Lpc Fácán sor 25, Gödöllö, H-2100, Hungary

Abbreviations: CMS: Cardiometabolic Syndrome; BPHi: Blood Pressure-to-Height Index; BP: Blood Pressure; SBP: Systolic Blood Pressure; DBP: Diastolic Blood Pressure; CA: Cerebral Autoregulation

\section{Introduction}

Medical doctors due to economic considerations of the pharmaindustry and the flexibility of the human organization prescribe medicines with certain approximation. No patient will treated with daily 4 or $6 \mathrm{mg}$ enalapril, but with the available $5 \mathrm{mg}$ tablets. And the patient, independently of his/her actual age, height or weight, will reach the targeted blood pressure value. If not, dose will be increased according to the scale of dosage forms of the particular main ingredient. Contrarily, for an anaesthesiologists, who has to dosage the anaesthetics according to the actual body weight, would some inaccuracy of body weight make problem. However, in this case the anaesthesiologist has to consider the obesity, maybe based on the patients body mass index (BMI) as well because of the different pharmacokinetic parameters of the drugs in fat mass and lean body mass [1]. In this particular situation the BMI becomes an important factor.

\section{About the BMI}

BMI is an indirect measure of body fat based on the patients' weight in relation to height. The BMI has very different importance in the medicine. Firstly taken the above mentioned example, in case of wrong BMI value maybe the patient will wake up during the operation even if the dose per weight was right. Secondly BMI is a very different problem for the general practitioner, whose patient has an actual body weight but 1 month later he or she has an other one therefore the BMI should be taken just as a indicator of tendency. It is obvious that overweight and obese people should be advised toward more exercise and reasonable diet but the direction of counselling does not depend on the actual 2 pounds more or less but depends on the tendency. Thirdly the diagnosis of malnutrition may be associated with serious clinical outcomes [2], and the BMI plays in the age-group of children over 2 years or hospitalized patients over
65 years an important role. In general, BMI is a good measure on population level yet has limitations, thus they can usually be used for reference only. No doubt, BMI in the normal value is associated with the lowest risk of mortality, to this end it is beacon-light in the healtcare. Commonly used cutoff values of the BMI classification, however, must not be taken as only determinant data for relevant decisions. There are usually several other factors to be considered, too. Should anybody give serious advise for his/her patient on risk factors or therapeutic interventions, should based the message on the patients heath status and body composition (BIA, DEXA, MRI, etc.) not on BMI or other anthropometric formulas like inverse BMI, Broca-index or WheiR (Waist-height Ratio). With the hackneyed BMI there are a lot of problems. Often does not fits with height, age, sex, etc. Also the self-reporting is unreliable because some people over- or underestimate their weight and height $[3,4]$ others tell the truth [5], moreover BMI in childhood and adolescents can also be misleading. But ideal body weight calculations are not better either, especially the older equations [6]. We see, the best solution is the doctors personal judgement based on experience and professional skill.

\section{The Problematics of the Height and Blood Pressure (BP) Relationship}

This issue was intensively discussed in the past, especially by far-east researchers. Cardiometabolic syndrome (CMS) became an epidemy, nearly one third of the population of developed countries suffer from the disease. Due to the serious consequences of the CMS like complications of diabetes mellitus, ischemic and nonischemic myocardial dysfunction and heart failure, etc. healthcare professionals should help discover its components (hypertension, insulin resistance, dyslipidemia and obesity) as early as possible. One of the aids is the above mentioned BMI. Today, some other 
efficient tools to identify childhood hypertension are the blood pressure-to-height index (BPHi) [7] and the height-specific blood pressure threshold (HBP) [8]. But there is an other aspect of height and blood pressure (BP) ratio. In the short communication of JV Roth [9] we recently read that the "extra work on the heart required to maintain a higher blood pressure, that may just be the cost that must be paid for being tall". This means tall people are disadvantaged to shorter ones from hypertension point of view.

This is very exciting statement. The regulation of blood pressure is very complicated and cardiac output as well as blood vessel elasticity and peripheral resistance play an equally important role in it. All of them work under neurohumoral regulation. For the time being weare able to influence just separate mechanisms in the course of pharmacotherapy. It means we have separate agents to influence cardiac output and agents influencing the vascular resistance. But after every separate interventions central and peripheral blood pressure change in a harmonized manner. Patients can lie down and rise up without any orthostatic tension problem, heart, brain and all other organs are supplied with blood, it means the local blood pressure can be maintained everywhere. Postnatal increase of systolic blood pressure according to the percentiles finishes in girls earlier than in boys and stable " final BP " is usually reached by the end of first year of life. It is around 105/68 and 110/73 mmHg at $90 \%$ and $95 \%$ percentiles respectively. Afterwards slight increase in line with age and height is normal but significant increase in BP (> 2-3 mmHg / year or $5 \mathrm{~cm}$ in height) is not healthy [10]. According to the various pediatric guidelines $\mathrm{BP}>120 / 80 \mathrm{mmHg}$ is the cutoff line for diagnosis of prehypertension.

The heart is growing together with other organs but blood pressure under healthy conditions does not increases with the height or weight parallely. Oversimplified, the heart functions serve the childrens growth ie. the greater the demand for development is, the higher the [blood volume $\mathrm{X}$ cardiac output] product in order to supply cells and tissues with oxygen and substrates to the anabolism. The size and function of the heart always fit to the developmental phase. If growth stoped at $156 \mathrm{~cm}$ or $198 \mathrm{~cm}$ height, development of the heart follows this. And in this case the heart has no extra load to serv the demand of the taller persons. The functions of human body can not be described with pure physical rules eg. cardiovascular system is not like a network of rigid tubes. Should develop a continuously overload increased workload would make heart exhausted but according to my knowledge tall people are not more at risk for cardiomyopathy or chronic (congestive) heart failure, than short ones. In contrast, B Bourgeois and co-workers [11] reveald in a large and diverse cohort of patients negative correlation with height and systolic blood pressure (SBP) or height and puls pressure, while positive is the correlation between height and diastolic blood pressure (DBP).

Others also support the inverse association between height and risk of developing ischemic heart disease and stroke. Should the height difference make an extra load to the heart, would result in myocyte hypertrophy and/or increased wall-thickness. In the scientific literature I did not find any experience, that specifically referred to such impairment in tall people. In contrast hypertensive patients' heart produce these alterations [12] independently from height, which could be reversed - under experimental conditions - with therapeutic vasodilatation by angiotensin-II type 1 receptor blockers or, in lesser extent with ACE inhibitors [13]. Due to the successfull treatment options, confirmed hypertension can easily be normalized and prevalence of subsequent heart failure decreased significantly. Using 50 year's data of the Framingham study Levy reported a fall of CHF-mortality in men from $70 \%$ to $59 \%$ and in women $57 \%$ to $45 \%$ due to modern therapeutic interventions [14].

And what about the circulation in brain of tall people? As the brain is one of the most metabolically active organs, it is highly susceptible to changes in blood pressure. The cerebral autoregulation (CA) handles well hypertensive as well as hypotensive conditions. CA is still not fully understood but it plays a dominant role in cerebral blood flow homeostasis [15]. Cerebral circulation is extremely well adapted to protect the brain from hypertensive overperfusion. The atificial intervention by antihypertensive therapy may produce systemic hypotension which in turn activates counter-regulation in the brain. If hypotensive therapy causes BP $<130 / 80 \mathrm{mmHg}$ in the systemic arterial pressure the cerebral blood flow increses [16]. With chronic hypotension, however, the related brain cerebral hypoperfusion may cause cognitive deficits. Nevertheless one can not state that tall people have more often or more serious cognitive deficits than short ones.

\section{Conclusion}

The ratios of various physiological and pathophysiological parameters have their own place in the medicine. The body mass index is a measure that must be estimated prudently because BMI does not represent fat mass exactly. The limitations of such anthropometric equation are known by all medical professionals. The ratio of blood pressure and height is mostly used as a simple and reliable indicator for juvenile prehypertension and hypertension. In adults this ratio has not top priority in decision-making about interventions to improve risk factors of metabolic syndrome including hypertension.

\section{References}

1. Telessy IG, Huttar HS (2017) Obesity related alterations in pharmacokinetics and pharmacodynamics of drugs: emerging clinical imolications in obese patients - Part II. Adipobiology 9: 39-48.

2. Chen MY, Yang YJ (2018) Being underweight is an inependent risk factor for poor putcomea among acutely critically ill children. Nutr Clin Pract 33(3): 433-438.

3. Gavriilidou NN, Pihlsgard M, Elmstahl S (2015) High degree of BMI misclassification of malnutrition among Swedish elderly population: age-adjusted height estimation using knee height and drmispan. Eur J Clin Nutr 69(5): 565-571.

4. Gildner TE, Barrett TM, Liebert MA (2015) Does BMI generated by selfreported height and weight measure $u$ pin older adults from middleincome countries? Results from the study on global AGEing and adult health (SAGE). BMC Obesity 2: 44.

5. Poston WS, Jitnarin N, Haddock CK (2014) Accuracy of self-reported weight, height and BMI in US firefighters. Occup Med (Lond) 64(4): 246254 .

6. Peterson CM, Thomas DM, Blackburn GL, Heymsfield S (2016) Universal equation for estimating ideal body weight and body weight at any BMI. 


\section{Am J Clin Nutr 103(5): 1197-1203.}

7. Yin X, Liu Q Bovet P (2016) Performance of blood pressure-to-height ratio as a screening tool for elevated blood pressure in pediatric population: a systematic meta-analysis. J Hum Hypertens 30(11): 697702.

8. Di Bonito P, Valerio G, Pacifico L (2017) A new index to simplify the screening of hypertension in overweight or obese youth. Nutr Metab cardiovasc Dis 27(9): 830-835.

9. Roth JV (2018) Taller people should have higher MBI's and blood pressure measurements as their normal. Biomed J Sci Tech Res 6(4): BJSTR MS ID 001381.

10. Flynn JT, Daniels SR, Hayman LL (2014) Update: Ambulatory blood pressure monitoring in children and adolescents. Hypertension 63(5): 1116-1135

11. Bourgeois B, Watts K, Thomas DM (2017): Association between height and blood pressure int he Unites States population. Medicine (Baltimore) 96(50): e9233.

\section{ISSN: 2574-1241}

DOI: 10.26717/BJSTR.2018.07.001505

Istvan G Telessy. Biomed J Sci \& Tech Res

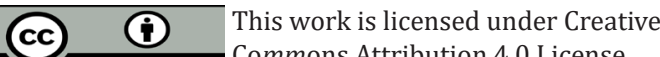

Submission Link: https://biomedres.us/submit-manuscript.php
12. Ye Chen Izu, Ling Chen, Bányász T (2007) Hypertension-induced remodelling of cardiac excitation-contraction coupling in ventricular myocytes occurs prior to hypertrophy development. Am J Physiol Heart Circ Physiol 293(3): H3301-H3310.

13. Tamura T, Said S, Harris L (2000) Reverse remodellingof cardiac myocyte hypertrophy in hypertension and failure by tartgeting of the renin-angiotensin system. Circulation 102(2): 253-259.

14. Levy D, Kechaiah S, Larson MG (2002) Long-term trend sin the incidence of and survival with heart failure. N Eng J Med 347(18): 1397-1402.

15. Tzeng YC, Ainslie PN (2014) Blood pressure regulation IX: cerebral autoregulation inder blood pressure challenges. Eur J Appl Physiol 114(3): 545-559.

16. Tryambake D, He J, Firbank MJ (2013) Intensive blood pressure lowering increases cerebral blood flow in older subjects with hypertension. Hypertension 61(6): 1309-1315.

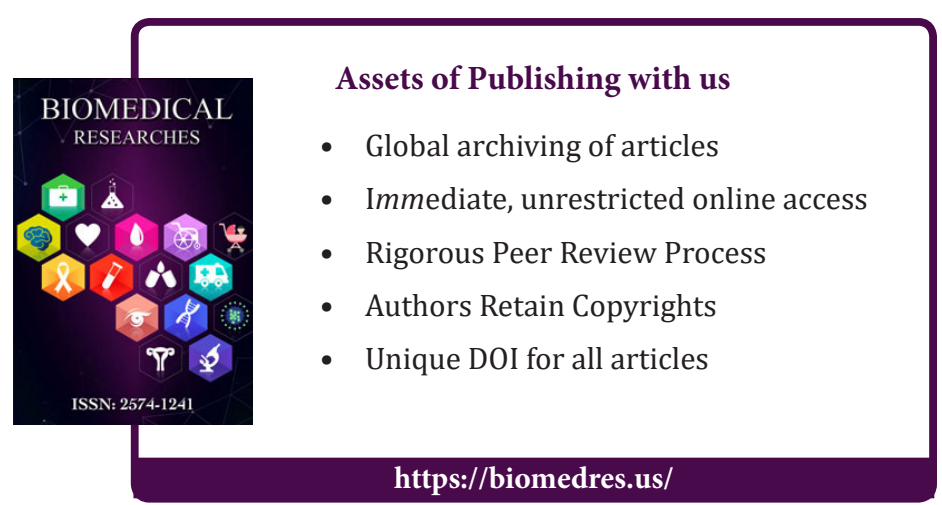

